\title{
Actividad física, aspectos sociodemográficos, familiares, hábitos saludables y atención en salud de adultos mayores
}

\author{
Physical activity level, sociodemographic and family aspects, healthy habits and health care for older adults \\ Mauricio Hernández-Carrillo1,2 orcid.org/0000-0001-8816-7726 \\ Carlos Andrés Quiroz-Mora1,3 orcid.org/0000-0003-4106-3019 \\ Jhonatan Betancourt-Peña1,4* orcid.org/0000-0002-7292-7628 \\ 1. Facultad de Salud y Rehabilitación, Institución Universitaria Escuela Nacional del Deporte. Cali, Colombia. \\ 2. Grupo de Investigación en Estudios de Salud y Sociedad (GIESS), Institución Universitaria Escuela Nacional del Deporte. Cali, \\ Colombia. \\ 3. Grupo de Investigación en Actividad Física y Salud (GIAF), Universitaria Escuela Nacional del Deporte. Cali, Colombia. \\ 4. Facultad de Salud, Universidad del Valle.
}

Hernández-Carrillo M, Quiroz-Mora CA, Betancourt-Peña, J. Nivel de actividad física, aspectos sociodemográficos, familiares, hábitos saludables y atención en salud de adultos mayores. Univ. Salud. 2021;23(3):263-271. DOI: https://doi.org/10.22267/rus.212303.240

\section{Resumen}

Introducción: La práctica de la actividad física en el adulto mayor tiene influencia de tipo social, cultural y demográfica. Objetivo: Comparar la relación entre el nivel de actividad física informada en un grupo de adultos mayores y sus características: sociodemográficas, familiares, de hábitos saludables, enfermedad y de la atención en salud. Materiales y métodos: Estudio observacional-transversal en población de adultos mayores de Cali. Se realizó un muestreo simple estandarizado. La actividad física se midió mediante Cuestionario Internacional de Actividad Física (IPAQ) comparando grupos en actividad física intensa-moderada (AFIM) y actividad física baja-inactiva (AFBI). Resultados: Se vincularon 189 participantes AFIM y 364 para AFBI. Se obtuvieron relaciones estadísticamente significativas en las variables zona de vivienda, ocupación, fuente de ingresos y dependencia $(p=0,000)$; composición familiar $(p=0,008)$, estar solo $(p=0,031)$, red social $(p=0,000)$, consumo de alcohol, ejercicio, hipertensión arterial, consumo de fármacos $(p=0,000)$, diabetes mellitus $(p=0,045)$, índice de masa corporal $(p=0,032)$, afiliación, distancia del centro de atención y costos $(p=0,000)$. Conclusiones: Los hábitos como el consumo de alcohol y tabaco, con enfermedades como la hipertensión arterial y la diabetes mellitus se relacionaron con nivel de AFBI. Los adultos mayores que residen en la zona rural mostraron mayores niveles de actividad.

Palabras clave: Ejercicio físico; dinámica poblacional; relaciones familiares; hábitos; atención ambulatoria; anciano. (Fuente: DeCS, Bireme).

\begin{abstract}
Introduction: The practice of physical activity in the elderly is affected by social, cultural and demographic aspects. Objective: To compare the relationship between physical activity level and various population and medical characteristics (sociodemographic, family, healthy habits, illnesses, and health care) in a group of older adults. Materials and methods: An observational crosssectional study was conducted with an elderly population from Cali (Colombia), using a simple standardized sampling. The International Physical Activity Questionnaire (IPAQ) was used to assess physical activity, comparing groups with intense-moderate physical activity (IMPA) and those showing low-inactive physical activity (LIPA). Results: The study included 189 and 364 patients with IMPA and LIPA, respectively. Statistically significant differences were found for the variables: housing area, employment, source of income and dependency ( $p=0.000)$; family composition $(p=0.008)$, living alone $(p=0.031)$, social network $(p=0.000)$, alcohol consumption, exercise, high blood pressure, medication use $(p=0.000)$, diabetes mellitus $(p=0.045)$, body mass index $(p=0.032)$, health insurance affiliation, distance from health care center, and costs $(p=0.000)$. Conclusions: Habits such as alcohol and tobacco consumption together with diseases such as high blood pressure and diabetes mellitus are related to LIPA. Also, older adults residing in rural areas showed higher activity levels.
\end{abstract}

Keywords: Exercise; population dynamics; family relations; habits; ambulatory care; aged. (Source: DeCS, Bireme).

*Autor de correspondencia

Jhonatan Betancourt Peña

e-mail: johnnatanbp@hotmail.com 


\section{Introducción}

En Colombia, se ha evidenciado que el envejecimiento en la población de mayores de 65 años, tiene las más altas tasas de crecimiento comparadas con los otros grupos etarios; para el año 2013 se estimó que este grupo de personas era de 3.815.453, incluso para el 2015 el crecimiento de la población mayor era de un 3,51\% y al 2018 representa el 9,1\% de la población total(1).

Junto con el proceso de envejecimiento, la práctica de actividad física en los adultos mayores se ve disminuida por los cambios en la salud, ya que se incrementan las enfermedades crónicas no transmisibles y con esto el consumo de los fármacos; además, se presentan cambios en su entorno social, ocupacional, influidos por un incremento en la necesidad de asistencia sanitaria(2-4). Los hábitos como el consumo de tabaco y alcohol en algún momento de la vida han demostrado una relación con un bajo nivel de actividad física, así como las prácticas de actividad física a temprana edad han mostrado perpetuarse durante el resto de la vida(5-7).

En el adulto mayor, el deterioro funcional es uno de los principales factores que influyen en la disminución de la calidad de vida, ocasionando efectos deletéreos en su estado de salud(3), que conllevan a una pérdida importante de la actividad física regular en las actividades de la vida diaria y básicas cotidianas(4). La realización constante de actividad física en este grupo de interés favorece la disminución de los efectos osteomusculares del envejecimiento como la sarcopenia y la osteoporosis(5), además cambios sistémicos importantes ocasionadas por la Hipertensión Arterial (HTA) y la Diabetes Mellitus (DM) ${ }^{(8)}$.

Si bien, los beneficios de la actividad física son conocidos, los adultos mayores desempeñan en su mayoría actividades del hogar y son físicamente activos para chequeos habituales de salud, siendo sedentarios generalmente la mayor parte del tiempo debido al cambio de las dinámicas del curso de la vida relacionados con el trabajo, la familia, la jubilación y su círculo social $(9,10)$. También se ha identificado que la práctica de la actividad física en el adulto mayor está relacionada representativamente en las mujeres, para quienes se evidencia un mejor control de enfermedades y hábitos de vida saludables(10).
Según algunos autores, los factores demográficos, familiares, del entorno social y del sistema de salud han mostrado una relación estrecha con los niveles de actividad física informada por los adultos mayores(11), incluso se ha descrito esta situación en diversas regiones del Colombia(12). También se ha demostrado una relación importante de la urbanización, la economía, y el círculo familiar sobre el sedentarismo ${ }^{(9,10)}$.

No se han realizado análisis según el nivel de actividad física en adultos mayores tanto de la zona rural (corregimientos, veredas, campo) como en la zona urbana (ciudad, comunas, barrios). El bajo tamaño de los grupos poblacionales para el análisis en los estudios de referencia no permite una comprensión representativa del fenómeno. Por lo expuesto, este estudio tuvo como finalidad comparar la relación entre el nivel de actividad física informada en un grupo de adultos mayores y sus características: sociodemográficas, familiares, de hábitos saludables, enfermedad y de la atención en salud.

\section{Materiales y métodos}

Se realizó un estudio observacional de corte transversal. La población estuvo constituida por adultos mayores vinculados en el año 2019 a dos redes de salud del municipio, una red perteneciente a la zona rural y otra a la zona urbana de la ciudad de Cali. La selección de la población se realizó por intensión y mediante un muestreo simple estandarizado por edad con un nivel de confianza al $95 \%$ y un margen de error de 5\%. Finalmente, se vincularon 553 participantes entre los meses de febrero a abril del 2019 y correspondían a los registros que reposaban en la base de datos de las redes de salud.

Como criterios de inclusión se tuvieron en cuenta: adultos mayores de 60 años residentes al menos un año en la ciudad, con capacidad para participar de forma independiente en el estudio, como criterios de exclusión: presentar una enfermedad descompensada que limitara la realización de cuestionarios y actividad física. Las dimensiones analizadas en este estudio fueron: factores sociodemográficos, familiares, hábitos, salud y el sistema. Las variables de la dimensión sociodemográfica y familiar fueron: zona de vivienda, sexo, edad, estado civil, nivel de estudios, ocupación, fuente de ingresos, dependencia económica, composición familiar, estar solo en el día, disponer de 
un cuidador personal, pertenecer a una red social o comunitaria. Las variables de los factores relacionados con los hábitos, la salud y el sistema fueron: hábito de consumo de alcohol, tabaco, fármacos, hábito de ejercicio físico, HTA, DM, clasificación del Índice de Masa Corporal (IMC), afiliación a salud, percepción de distancia al centro médico, costos para acceder al servicio.

Las dimensiones y las variables fueron evaluadas mediante aplicación de un cuestionario estructurado a través de entrevista directa. El nivel de actividad física se evaluó mediante el cuestionario Internacional de Actividad Física (IPAQ), que mide la actividad física en índice metabólico (Mets) teniendo en cuenta las actividades realizadas durante los últimos 7 días, categorizando el nivel en intensa, moderada, bajo o inactivo(11,13). Se establecieron 2 puntos de corte para determinar la población con Actividad Física Intensa Moderada (AFIM) $(n=189)$ y Actividad Física Baja inactiva (AFBI) $(n=364)^{(11-14)}$.

Las encuestas y mediciones fueron realizadas por profesionales de la salud previamente entrenados en caracterización de población adulta mayor y en toma de talla y peso. Previamente los adultos mayores que voluntariamente aceptaron participar del estudio fueron contactados vía telefónica y citados a una institución prestadora de servicios de salud de la red de salud de la ciudad de Cali, en la cual firmaron el consentimiento informado. Posteriormente, se procedió a la toma del peso, usando balanza digital calibrada y la talla por medio de estadiómetro de precisión SECA para luego obtener el IMC [peso (kg)/talla $\left.\left(\mathrm{m}^{2}\right)\right]$. Luego se realizó el cuestionario de recolección de información a cada adulto mayor contemplando una duración de 20 minutos para cada uno.

\section{Análisis estadístico}

Se realizó comparación de los factores sociodemográficos, familiares, hábitos saludables y de atención en salud, entre los participantes clasificados con AFMI y AFBI, estableciendo relación estadística con la prueba Chi-cuadrado de Pearson. Se utilizó Excel para la elaboración de los cuadros y el software SPSS versión 25 para la obtención de estadísticos de prueba. La normalidad se evaluó utilizando la prueba de Kolmogórov-Smirnov y para comparar de medianas se realizó diagrama de cajas. Se utilizó la prueba $U$ de Mann Whitney para muestras independientes.

\section{Consideraciones éticas}

El estudio fue aprobado por el Comité de Ética Humana y por la red de salud de la ciudad de Cali Acta (200.3.2.180.2016), por la cual se adoptaron todas las recomendaciones de las normas Helsinki y la resolución 008430 del Ministerio de Salud de Colombia; su vez, todos los participantes conocían los alcances del estudio, decidieron hacer parte del estudio de manera voluntaria y firmaron el consentimiento informado.

\section{Resultados}

De los 553 adultos mayores participantes del estudio, $15 \%$ eran hombres y el $85 \%$ restante mujeres con edades entre 50 y 95 años, con un promedio de 67,22 $\pm 8,9$ (Figura 1). El 51,5\% tenía como residencia zona urbana y el $48,5 \%$ zona rural, el grado de escolaridad representativo fue "primaria" para un $60 \%$ de la población. A nivel familiar, el $32 \%$ de los participantes son solteros, el $68 \%$ tiene labores del hogar como principal ocupación y el $50 \%$ vive con sus familiares, el 68\% está acompañado la mayor parte del tiempo a pesar de que el $29 \%$ prefiere estar en solo (Tabla 1).

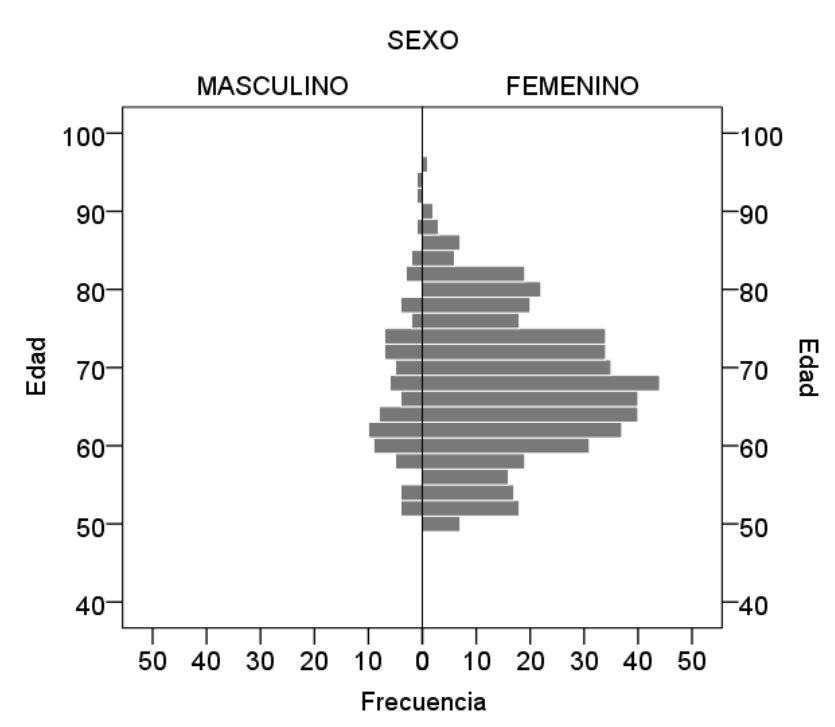

Figura 1. Pirámide poblacional de Adultos participantes residentes en Cali-Colombia 2019

En cuanto a la medición del IMC el 65\% de las personas se encontraron en condición de sobrepeso y obesidad, el 39\% consume o ha consumido alcohol, el $24 \%$ fuma o ha fumado en algún momento, y el $61 \%$ realiza ejercicio físico. El $80 \%$ de la población padece HTA y $23 \%$ DM, y un 90\% de ellas tienen prescripción farmacológica. Para la variable atención en salud predominó en un $65 \%$ el régimen subsidiado y un 
$30 \%$ de los adultos mayores percibe que su centro médico de atención es lejano (Tabla 2).

Se realizó prueba Kolmogórov-Smirnov para evaluar normalidad en la variable edad en ambos grupos: $\operatorname{AFIM}(p=0,026)$ y AFBI $(p=0,004)$, al no ajustarse a la normal se utilizó la mediana para compararlos. La mediana de los adultos mayores que realizan AFIM fue de 65 años (59 - 72), por debajo de la mediana de los que realizan AFBI: 67 años (62 - 74). La prueba $U$ de Mann Whitney evidenció que no existen diferencias estadísticamente significativas por grupo etario $(p=0,106)$, entre los niveles de actividad física en los adultos mayores.

Tabla 1. Comparación de las características sociodemográficas y familiares según el nivel de actividad física en adultos mayores

\begin{tabular}{|c|c|c|c|c|c|c|c|c|}
\hline \multirow{2}{*}{ Variable } & \multirow{2}{*}{ Categoría } & \multicolumn{2}{|c|}{ Total } & \multicolumn{2}{|c|}{ AFIM } & \multicolumn{2}{|c|}{ AFBI } & \multirow{2}{*}{ Valor $p$} \\
\hline & & $n=553$ & $\%$ & $n=189$ & $\%$ & $n=364$ & $\%$ & \\
\hline \multirow[t]{2}{*}{ Zona de Vivienda } & Urbana & 285 & 51,5 & 21 & 11,1 & 264 & 72,5 & $<0,0001^{*}$ \\
\hline & Rural & 268 & 48,5 & 168 & 88,9 & 100 & 27,5 & \\
\hline \multirow[t]{2}{*}{ Sexo } & Masculino & 83 & 15,0 & 26 & 13,8 & 57 & 15,7 & 0,552 \\
\hline & Femenino & 470 & 85,0 & 163 & 86,2 & 307 & 84,3 & \\
\hline \multirow[t]{4}{*}{ Etnia Auto percibida } & Mestizo & 392 & 70,9 & 139 & 73,5 & 253 & 69,5 & $0,020^{*}$ \\
\hline & Blanco & 60 & 10,8 & 26 & 13,8 & 34 & 9,3 & \\
\hline & Afrodescendiente & 80 & 14,5 & 16 & 8,5 & 64 & 17,6 & \\
\hline & Indígena & 21 & 3,8 & 8 & 4,2 & 13 & 3,6 & \\
\hline \multirow[t]{4}{*}{ Estado civil } & Soltero & 179 & 32,4 & 52 & 27,5 & 127 & 34,9 & 0,085 \\
\hline & Casado & 174 & 31,5 & 72 & 38,1 & 102 & 28 & \\
\hline & Divorciado & 54 & 9,8 & 16 & 8,5 & 38 & 10,4 & \\
\hline & Viudo & 146 & 26,4 & 49 & 25,9 & 97 & 26,6 & \\
\hline \multirow[t]{6}{*}{ Nivel de estudios } & Sin estudios & 60 & 10,8 & 21 & 11,1 & 39 & 10,7 & 0,31 \\
\hline & Primaria & 337 & 60,9 & 110 & 58,2 & 227 & 62,4 & \\
\hline & Secundaria & 113 & 20,4 & 37 & 19,6 & 76 & 20,9 & \\
\hline & Técnico & 28 & 5,1 & 12 & 6,3 & 16 & 4,4 & \\
\hline & Pregrado & 13 & 2,4 & 8 & 4,2 & 5 & 1,4 & \\
\hline & Posgrado & 2 & 0,4 & 1 & 0,5 & 1 & 0,3 & \\
\hline \multirow[t]{7}{*}{ Ocupación } & Hogar & 376 & 68,0 & 108 & 57,1 & 268 & 73,6 & $<0,0001^{*}$ \\
\hline & Independiente & 85 & 15,4 & 31 & 16,4 & 54 & 14,8 & \\
\hline & Pensionado & 50 & 9,0 & 28 & 14,8 & 22 & 6 & \\
\hline & Desempleado & 19 & 3,4 & 8 & 4,2 & 11 & 3 & \\
\hline & Dependiente & 18 & 3,3 & 10 & 5,3 & 8 & 2,2 & \\
\hline & Estudiante & 1 & 0,2 & 1 & 0,5 & 0 & 0 & \\
\hline & Comerciante & 4 & 0,7 & 3 & 1,6 & 1 & 0,3 & \\
\hline \multirow[t]{3}{*}{ Fuente de los ingresos } & Familiar & 324 & 58,6 & 87 & 46 & 237 & 65,1 & $<0,0001^{*}$ \\
\hline & Personal & 202 & 36,5 & 89 & 47,1 & 113 & 31 & \\
\hline & Subsidio & 27 & 4,9 & 13 & 6,9 & 14 & 3,8 & \\
\hline \multirow{2}{*}{$\begin{array}{l}\text { Dependencia } \\
\text { económica }\end{array}$} & $\mathrm{Si}$ & 346 & 62,6 & 98 & 51,9 & 248 & 68,1 & $<0,0001^{*}$ \\
\hline & No & 207 & 37,4 & 91 & 48,1 & 116 & 31,9 & \\
\hline \multirow{5}{*}{ Composición Familiar } & Vive solo & 70 & 12,7 & 23 & 12,2 & 47 & 12,9 & $0,008^{*}$ \\
\hline & Vive solo con su pareja & 72 & 13,0 & 35 & 18,5 & 37 & 10,2 & \\
\hline & Vive con pareja e hijos & 65 & 11,8 & 29 & 15,3 & 36 & 9,9 & \\
\hline & Vive con pareja y parientes & 66 & 11,9 & 22 & 11,6 & 44 & 12,1 & \\
\hline & Sin pareja, vive con familiares & 280 & 50,6 & 80 & 42,3 & 200 & 54,9 & \\
\hline \multirow[t]{2}{*}{ Solo en el día } & Nunca - a ratos & 380 & 68,8 & 126 & 66,6 & 254 & 69,8 & $0,031^{*}$ \\
\hline & Medio día - siempre & 173 & 31,3 & 63 & 33,3 & 110 & 30,2 & \\
\hline \multirow[t]{2}{*}{ Cuidador personal } & $\mathrm{Si}$ & 31 & 5,6 & 11 & 5,8 & 20 & 5,5 & 0,875 \\
\hline & No & 522 & 94,4 & 178 & 94,2 & 344 & 94,5 & \\
\hline Red $\quad$ social & Ninguna, prefiere estar solo & 159 & 28,8 & 24 & 12,7 & 135 & 37,1 & $<0,0001^{*}$ \\
\hline \multirow[t]{5}{*}{ comunitaria } & Amistades & 24 & 4,3 & 7 & 3,7 & 17 & 4,7 & \\
\hline & Familia & 53 & 9,6 & 30 & 15,9 & 23 & 6,3 & \\
\hline & Comunitarias o del barrio & 92 & 16,6 & 57 & 30,2 & 35 & 9,6 & \\
\hline & Trabajo o estudio & 2 & 0,4 & 1 & 0,5 & 1 & 0,3 & \\
\hline & Grupo especial & 223 & 40,3 & 70 & 37,0 & 153 & 42 & \\
\hline
\end{tabular}

*Significativos al 0,05 AFIM: Actividad Física Intensa Moderada AFBI: Actividad Física Baja inactiva 
De los 553 adultos mayores evaluados, 189 se encontraron en nivel AFIM y 364 en nivel AFBI. Con respecto a las características sociodemográficas y familiares, comparadas entre quienes obtuvieron un nivel de AFIM con los que tenían un nivel AFBI, se obtuvo que en 8 variables hubo diferencias estadísticamente significativas: zona de vivienda $(p<0,0001)$, etnia auto percibida $(p=0,020)$, ocupación $(p<0,0001)$, fuente de ingresos $(p<0,0001)$, dependencia económica $(p<0,0001)$, composición familiar $(p=0,008)$, estar solo durante el día $(p=0,031)$, red social o comunitaria $(p<0,0001)$ (Tabla 1).

Para los adultos mayores que se clasificaron en el nivel AFIM, los factores que tuvieron mayor representación fueron; residir en la zona rural $(88,9 \%)$, auto percibirse mestizo $(73,5 \%)$ y blanco
$(13,8 \%)$, ser pensionado $(14,8 \%)$ o estar laborando de forma independiente (18\%), tener independencia económica $(48,1 \%)$, convivir en familia $(15,9 \%)$ y pertenecer a grupos sociales o comunitarios $(30,2 \%)$.

En cuanto al grupo que se clasificó en el nivel de AFBI, los factores que tuvieron mayor representación fueron: residir en zona urbana $(72,5 \%)$, auto percibirse afrodescendiente $(17,6 \%)$, ocupación relacionada con el hogar $(73,6 \%)$, tener una dependencia económica por la familia $(68,1 \%)$, no tener redes familiares y sociales $(37,1 \%)$.

Para los factores como el sexo, estado civil, nivel de estudios, composición familiar y requerimiento de cuidador personal, no existieron diferencias estadísticamente significativas entre los grupos de adultos mayores clasificados en AFMI y AFBI.

Tabla 2. Comparativo de las características relacionadas con los hábitos, la salud y la atención en salud según el nivel de actividad física en adultos mayores

\begin{tabular}{|c|c|c|c|c|c|c|c|c|}
\hline \multirow{2}{*}{ Variable } & \multirow{2}{*}{ Categoría } & \multicolumn{2}{|c|}{ Total } & \multicolumn{2}{|c|}{ AFIM } & \multicolumn{2}{|c|}{ AFBI } & \multirow{2}{*}{ Valor $p$} \\
\hline & & $n=553$ & $\%$ & $n=189$ & $\%$ & $n=364$ & $\%$ & \\
\hline \multirow{3}{*}{$\begin{array}{l}\text { Hábitos de } \\
\text { consumo de } \\
\text { alcohol }\end{array}$} & Nunca & 338 & 61,1 & 136 & 72 & 202 & 55,5 & $<0,0001^{*}$ \\
\hline & En el pasado & 167 & 30,2 & 37 & 19,6 & 130 & 35,7 & \\
\hline & Actualmente & 48 & 8,7 & 16 & 8,5 & 32 & 8,8 & \\
\hline \multirow{3}{*}{$\begin{array}{l}\text { Hábito de } \\
\text { consumo de } \\
\text { tabaco }\end{array}$} & Nunca & 429 & 77,6 & 156 & 82,5 & 273 & 75 & 0,124 \\
\hline & En el pasado & 111 & 20,1 & 29 & 15,3 & 82 & 22,5 & \\
\hline & Actualmente & 13 & 2,4 & 4 & 2,1 & 9 & 2,5 & \\
\hline \multirow{3}{*}{$\begin{array}{l}\text { Hábito de } \\
\text { ejercicio físico }\end{array}$} & Nunca & 126 & 22,8 & 25 & 13,2 & 101 & 27,7 & $<0,0001^{*}$ \\
\hline & En el pasado & 87 & 15,7 & 20 & 10,6 & 67 & 18,4 & \\
\hline & Actualmente & 340 & 61,5 & 144 & 76,2 & 196 & 53,8 & \\
\hline \multirow{2}{*}{$\begin{array}{l}\text { Hipertensión } \\
\text { arterial }\end{array}$} & Sin HTA & 109 & 19,7 & 57 & 30,2 & 52 & 14,3 & $<0,0001^{*}$ \\
\hline & Con HTA & 444 & 80,3 & 132 & 69,8 & 312 & 85,7 & \\
\hline \multirow{2}{*}{$\begin{array}{l}\text { Diabetes } \\
\text { mellitus }\end{array}$} & Sin DM & 426 & 77,0 & 155 & 82,0 & 271 & 74,5 & $0,045^{*}$ \\
\hline & Con DM & 127 & 23,0 & 34 & 18,0 & 93 & 25,5 & \\
\hline \multirow{2}{*}{$\begin{array}{l}\text { Consumo de } \\
\text { fármacos }\end{array}$} & $\mathrm{Si}$ & 498 & 90,1 & 155 & 82,0 & 343 & 94,2 & $<0,0001^{*}$ \\
\hline & No & 55 & 9,9 & 34 & 18,0 & 21 & 5,8 & \\
\hline \multirow{3}{*}{$\begin{array}{l}\text { Clasificación } \\
\text { IMC }\end{array}$} & Bajo peso & 7 & 1,3 & 0 & 0,0 & 7 & 1,9 & $0,032^{*}$ \\
\hline & Peso normal & 188 & 34,0 & 66 & 34,9 & 122 & 33,5 & \\
\hline & Sobrepeso y obesidad & 358 & 64,7 & 123 & 65,2 & 235 & 64,6 & \\
\hline \multirow{3}{*}{$\begin{array}{l}\text { Afiliación a } \\
\text { salud }\end{array}$} & Contributivo & 170 & 30,7 & 88 & 46,6 & 82 & 22,5 & $<0,0001^{*}$ \\
\hline & Subsidiado & 361 & 65,3 & 90 & 47,6 & 271 & 74,5 & \\
\hline & Población pobre no asegurada & 21 & 3,8 & 11 & 5,8 & 10 & 2,7 & \\
\hline \multirow{3}{*}{$\begin{array}{l}\text { Percepción de } \\
\text { distancia al } \\
\text { centro médico }\end{array}$} & Cerca & 278 & 50,3 & 72 & 38,1 & 206 & 56,6 & $<0,0001^{*}$ \\
\hline & Normal & 113 & 20,4 & 56 & 29,6 & 57 & 15,7 & \\
\hline & Lejos & 162 & 29,3 & 61 & 32,3 & 101 & 27,7 & \\
\hline \multirow{3}{*}{$\begin{array}{l}\text { Costos para } \\
\text { acceder al } \\
\text { servicio }\end{array}$} & Bajos & 385 & 69,6 & 100 & 52,9 & 285 & 78,3 & $<0,0001^{*}$ \\
\hline & Normal & 125 & 22,6 & 70 & 37 & 55 & 15,1 & \\
\hline & Altos & 43 & 7,8 & 19 & 10,1 & 24 & 6,6 & \\
\hline
\end{tabular}

*Significativos al 0,05 
Al comparar las características relacionadas con los hábitos y la atención en salud entre quienes obtuvieron un nivel de AFIM con los que tenían un nivel de AFBI, se obtuvo que en 9 variables hubo diferencias estadísticamente significativas: consumo de alcohol $(p<0,0001)$, ejercicio físico $(p<0,0001)$, HTA $(p<0,0001)$, DM $(p=0,045)$, consumo de fármacos $(p<0,0001)$, clasificación del IMC $(p=0,032)$, afiliación al sistema de salud $(p<0,0001)$, percepción de la distancia al centro médico $(p<0,0001)$, y costos para acceder a los servicios $(p<0,0001)$ (Tabla 2$)$.

Para los adultos mayores que se clasificaron en el nivel AFIM, los hábitos que tuvieron mayor representación fueron: no consumo de alcohol (72\%), no consumo de tabaco $(82,5 \%)$, realización de ejercicio físico en la actualidad (76,2\%), diagnóstico de HTA $(69,8 \%)$, consumo de fármacos (82\%). En cuanto al grupo que se clasificó en el nivel AFBI, los hábitos que tuvieron mayor representación fueron: consumo de alcohol en el pasado (35\%), consumo de tabaco en el pasado $(22,5 \%)$, nunca haber realizo ejercicio físico $(27,7 \%)$, tener HTA (85\%), y consumir fármacos $(94,2 \%)$.

Para el IMC a pesar de existir diferencias estadísticamente significativas $(p=0,032)$, los porcentajes fueron muy semejantes entre los dos grupos.

De forma general, los resultados muestran algunas variables con diferencias marcadas entre el grupo de AFMI y AFBI que llaman la atención, tales como: el área donde reside, el consumo de fármacos, la realización de ejercicio físico, antecedentes de HTA y DM, la dependencia económica y el consumo de alcohol y tabaco (Figura 2).

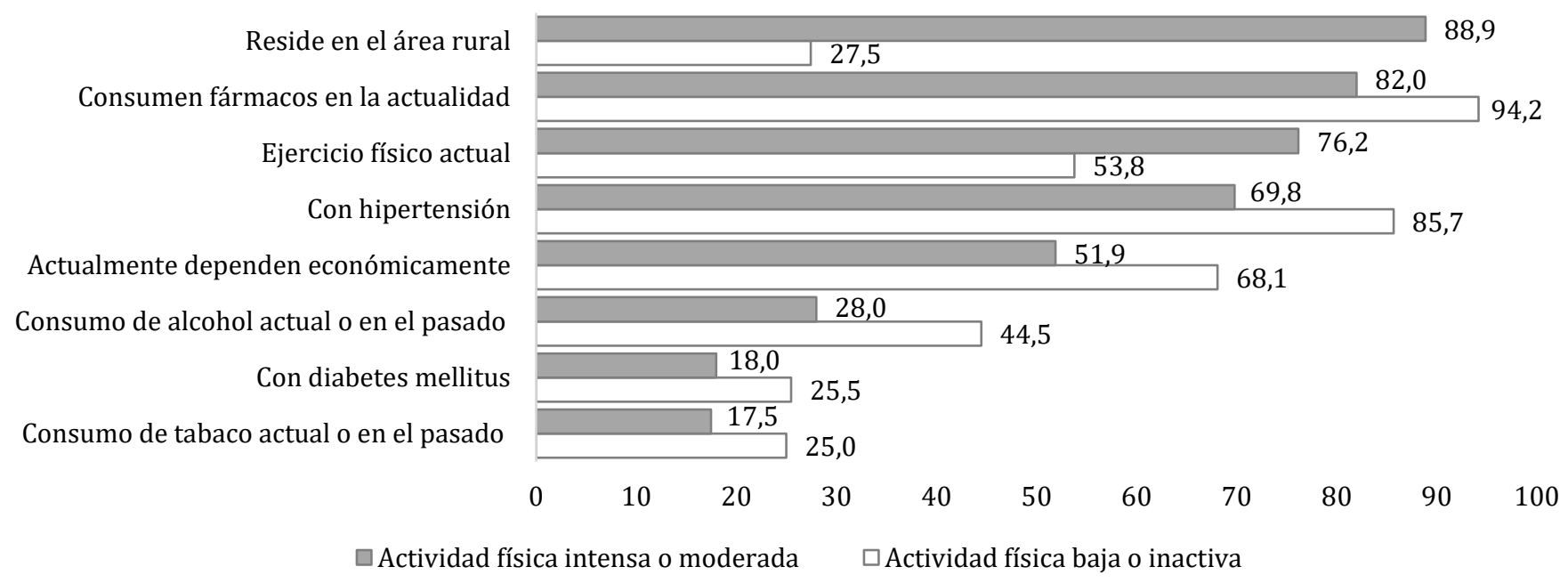

Figura 2. Principales diferencias en los adultos mayores con distintos niveles de actividad física

\section{Discusión}

La evaluación de la actividad física ha sido un aspecto determinante para la salud en los diversos escenarios sociales, culturales y políticos de las agendas mundiales(7,8,10). Su evaluación y en particular en el adulto mayor, requiere de instrumentos confiables y validados que permitan comprender con mayor profundidad una categorización en función del nivel de actividad física realizada por las personas. El IPAQ ha sido ampliamente utilizado para determinar la realización de la actividad física en Colombia, lo que permite la comparabilidad con las diferentes realidades sobre esta temática en el país(15,16). Igualmente, este cuestionario ha sido utilizado en otros países representando una de las formas más adecuadas para medir el nivel de actividad física en el adulto mayor a nivel mundial(17-20).

La práctica de la actividad física en los adultos mayores puede variar según los aspectos demográficos, culturales, sociales y según los sistemas de atención sanitaria, o también puede cambiar según el entorno rural o urbano en donde se 
ejecute(21-26). El nivel de actividad física para la población adulta mayor de esta investigación mostró una baja prevalencia $(34,2 \%)$, con respecto a otros estudios en Colombia que presentaron prevalencias adecuadas de actividad física de hasta un $50 \%(15,27)$.

La práctica de la actividad física en el adulto mayor fue más representativa en población femenina con edades cercanas a los 60 años. Este aspecto puede tener connotaciones epidemiológicas importantes como: la pirámide poblacional en Colombia con población femenina representativa en los últimos quinquenios, la práctica de actividad física vinculada a la participación dentro de los grupos de adultos mayores, donde se encuentra mayor población femenina, y la adherencia a la práctica de la actividad física es mayor en el sexo femenino(28). Con el incremento de la edad las prevalencias de sedentarismo y los niveles de personas con AFBI tienden a aumentar ${ }^{(29) .}$

Los hallazgos de esta investigación evidenciaron que los adultos mayores que residen en la zona rural, mostraron mayores niveles de actividad física comparado con los adultos de la zona urbana, esta diferencia podría relacionarse con aspectos estructurales del entorno y los diseños urbanos para la movilidad, puesto que las barreras arquitectónicas dificultan la práctica de actividad física, obligando a los adultos mayores a buscar espacios adaptados para la realización de ejercicio(23,24), muestra de ello, la práctica irregular en parques por grupos de adultos mayores.

Por otra parte, el espacio y el entorno juegan un papel importante en la realización de actividades motrices, por cuanto el adulto que reside en la zona rural requiere mayor desplazamiento para ejecutar actividades de la vida diaria en comparación con las personas que residen en la zona urbana(25), así pues, estos entornos rurales presentan menos barreras espaciales y exige un mayor desplazamiento, que favorece sustancialmente la movilidad.

Aspectos como el nivel educativo, tipo de ocupación y las condiciones familiares son representativos de la población colombiana debido a la transición social, política y económica del país, en esta investigación la mayor proporción de los adultos mayores alcanzaron un nivel de escolaridad básica y al ser más representativo el género femenino la ocupación más desempeñada es el hogar(1), lo cual podría suponer una mayor influencia sobre la práctica de actividad física, debido a que las personas que residen en áreas urbanas concentran en mayor proporción las tareas al interior del hogar(10).

Otro aspecto que mostró influencia sobre la práctica de la actividad física es la soledad y depender económicamente de sus familiares, demostrando que aquellos que quienes viven en estas condiciones, tienden a tener más bajas prácticas de actividad física(26). Lo anterior evidencia que la actividad física en el envejecimiento, es un fenómeno social complejo donde los factores socioculturales representan potencialidades y debilidades en los estilos de vida(26).

Los hábitos desarrollados en el curso de la vida representan una gran parte de los hábitos en el adulto

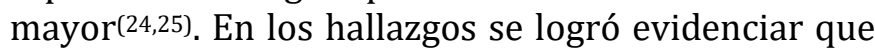
la población con niveles de AFIM realizaron más ejercicio físico en el pasado y en la actualidad; además presentaban menor consumo de alcohol y de tabaco. Para la población con nivel de AFBI presentaban mayor consumo de alcohol y de tabaco, y mayor prevalencia de enfermedades como HTA (85\%) y DM (25\%), con un consumo de fármacos del 94\%. La práctica de la actividad física regular es un factor protector para el desarrollo de enfermedades como la HTA y DM, así como los hábitos de consumo de alcohol y tabaco han demostrado estar asociados con niveles bajos de actividad física(30,31).

Por otra parte, el sistema de salud podría influir sustancialmente en la práctica de la actividad física(32). La población con nivel de AFBI presentó mayor vinculación en el régimen subsidiado de salud, que para el contexto colombiano es el mecanismo mediante el cual la población más pobre del país, sin capacidad de pago, tiene acceso a los servicios de salud a través de un subsidio que ofrece el Estado; a su vez, tienen mayor gasto de bolsillo para acceder a servicios de salud(33). Esto supone que, en Colombia, la realización de actividad física en el adulto mayor tiene un vínculo institucional con las entidades prestadoras de salud (EPS) a través de programas para la promoción de la salud y para el control de enfermedades crónicas no transmisibles, lo que supone la necesidad de pagos de cuotas moderadoras y de desplazamiento del adulto mayor a su centro médico de atención o a las instituciones prestadoras de servicio (IPS). Aun no se ha logrado una independencia del modelo institucionalizado de salud a un modelo de gestión del autocuidado(33). 
Vale la pena declarar que este estudio cuenta con limitaciones y que no es posible hacer inferencias sobre toda la población adulta mayor de Colombia debido a posibilidad de un sesgo en la selección y la mayor representatividad de mujeres. Sin embargo, se considera que se trata de una muestra significativa y es un punto de partida para programas de intervención en adultos mayores de la ciudad.

\section{Conclusión}

La práctica de AFIM se presentó en un 34\% de la población de estudio. El sexo femenino, la ocupación de hogar, la dependencia económica por parte de la familia y vivir solo, fueron las características sociodemográficas y familiares de mayor representatividad para ambos grupos. El nivel de AFBI se relacionó en mayor medida en la población que reside en el área urbana, con peores hábitos saludables como el consumo de alcohol y uso de tabaco y mayor predominio de enfermedades como la HTA y DM. Los adultos mayores que residen en la zona rural mostraron mayores niveles de actividad física y esta diferencia puede deberse con aspectos del entorno y a los diseños urbanos para la movilidad.

Conflicto de intereses: Ninguno declarado por los autores.

\section{Referencias}

1. Ministerio de Salud y Protección Social. Envejecimiento demográfico. Colombia 1951-2020. Dinámica demográfica y estructuras poblacionales [Internet]. Imprenta Nacional de Colombia; 2013. Disponible en: https://www.minsalud.gov.co/sites/rid/Lists/BibliotecaDi gital/RIDE/DE/PS/Envejecimiento-demografico-Colombia1951-2020.pdf

2. Singh L, Goel R, Rai RK, Singh PK. Socioeconomic inequality in functional deficiencies and chronic diseases among older Indian adults: a sex-stratified cross-sectional decomposition analysis. BMJ Open. 2019;9(2):1-9. DOI: 10.1136/bmjopen2018-022787.

3. Valderrama FP, Coronado FB, Aichele CV, Bravo EC. Nivel de actividad física y funcionalidad en adultos mayores. Cienc Act Física UCM. 2016;17(1):59-65. Disponible en: http://revistacaf.ucm.cl/article/view/91

4. Flynn MG, Markofski MM, Carrillo AE. Elevated Inflammatory Status and Increased Risk of Chronic Disease in Chronological Aging: Inflamm-aging or Inflamm-inactivity? Aging Dis. 2019;10(1):147-56. DOI: 10.14336\%2FAD.2018.0326.

5. Nguyen H, Chua K-C, Dregan A, Vitoratou S, Bayes-Marin I, Olaya B, et al. Factors Associated With Multimorbidity Patterns in Older Adults in England: Findings From the English Longitudinal Study of Aging (ELSA). J Aging Health. 2019;32(9):1120-32.

$10.1177 \% 2 \mathrm{~F} 0898264319891026$.
6. Schlesinger S, Neuenschwander M, Ballon A, Nöthlings U, Barbaresko J. Adherence to healthy lifestyles and incidence of diabetes and mortality among individuals with diabetes: a systematic review and meta-analysis of prospective studies. J Epidemiol Community Health. 2020;74(5):481-7. DOI: 10.1136/jech-2019-213415.

7. Ng R, Sutradhar R, Yao Z, Wodchis WP, Rosella LC. Smoking, drinking, diet and physical activity-modifiable lifestyle risk factors and their associations with age to first chronic disease. Int J Epidemiol. 2020;49(1):113-30. DOI: 10.1093/ije/dyz078.

8. Gichu M, Asiki G, Juma P, Kibachio J, Kyobutungi C, Ogola E. Prevalence and predictors of physical inactivity levels among Kenyan adults (18-69 years): an analysis of STEPS survey 2015. BMC public health. 2018;18(3):1217. DOI: 10.1186/s12889-018-6059-4.

9. Chong Daniel A. Aspectos biopsicosociales que inciden en la salud del adulto mayor. Rev Cuba Med Gen Integral. 2012;28(2):79-86.

http://scielo.sld.cu/scielo.php?pid=S086421252012000200009\&script=sci_arttext\&tlng=en

10. Zafra-Tanaka JH, Millones-Sánchez E, Retuerto-Montalvo MA. Factores sociodemográficos asociados a actividad física y sedentarismo en población peruana adulta. Rev Peru Epidemiol. http://hdl.handle.net/123456789/3133

11. Kaplan MS, Newsom JT, McFarland BH, Lu L. Demographic and psychosocial correlates of physical activity in late life. Am J Prev Med. 2001;21(4):306-12. DOI: 10.1016/S07493797(01)00364-6.

12. Liu H, Li F, Li J, Zhang Y. The relationships between urban parks, residents' physical activity, and mental health benefits: a case study from Beijing, China. J Environ Manage. 2017;190:223-30. DOI: 10.1016/j.jenvman.2016.12.058.

13. Sarmiento OL, Schmid TL, Parra DC, Díaz-del-Castillo A, Gómez LF, Pratt M, et al. Quality of Life, Physical Activity, and Built Environment Characteristics Among Colombian Adults. J Phys Act Health. 2010;7(Suppl 2):S181-95. DOI: 10.1123/jpah.7.s2.s181.

14. Craig CL, Marshall AL, Sjöström M, Bauman AE, Booth ML, Ainsworth BE, et al. International Physical Activity Questionnaire: 12-Country Reliability and Validity. Med Sci Sports Exerc. 2003;35(8):1381-95. DOI: 10.1249/01.MSS.0000078924.61453.FB

15. Gómez LF, Duperly J, Lucumí DI, Gámez R, Venegas AS. Nivel de actividad física global en la población adulta de Bogotá (Colombia): Prevalencia y factores asociados. Gac Sanit. 2005;19(3):206-13. Disponible en: https://www.scielosp.org/article/gs/2005.v19n3/206213/

16. Molina-González ME. Análisis del índice de masa corporal (IMC) y el nivel de actividad física en adultos mayores de la ciudad de barranquilla: un estudio correlacional. Biociencias. 2019;14(2):83-95. DOI: 10.18041/23900512/biociencias.2.6023.

17. Roszkowski A, Kulesza K, Cybulski M, Witkowska AM. Physical activity in type 1 diabetic young and early adults treated with insulin pump therapy. A preliminary report. Prog Health Sci. 2019;9(1):13-21. Disponible en: http://31.186.81.235:8080/api/files/view/934507.pdf

18. Castelli L, Galasso L, Mulè A, Bruno E, Shokohyar S, Esposito F, et al. Physical activity, chronotype and sleep in a sample of Italian elderly population. Sport Sci Health. 2020;16(1):5564. DOI: 10.1007/s11332-019-00573-x. 
19. Tomioka K, Iwamoto J, Saeki K, Okamoto N. Reliability and Validity of the International Physical Activity Questionnaire (IPAQ) in Elderly Adults: The Fujiwara-kyo Study. J Epidemiol. 2011;21(6):459-65. DOI: https://doi.org/10.2188/jea.JE20110003.

20. Ryan DJ, Wullems JA, Stebbings GK, Morse CI, Stewart CE, Onambele-Pearson GL. Reliability and validity of the international physical activity questionnaire compared to calibrated accelerometer cut-off points in the quantification of sedentary behaviour and physical activity in older adults. PloS one. 2018;13(4):e0195712. DOI: 10.1371\%2Fjournal.pone.0195712.

21. Scarapicchia TMF, Amireault S, Faulkner G, Sabiston CM. Social support and physical activity participation among healthy adults: a systematic review of prospective studies. Int Rev Sport Exerc Psychol. 2017;10(1):50-83. DOI: 10.1080/1750984X.2016.1183222.

22. Kepper MM, Myers CA, Denstel KD, Hunter RF, Guan W, Broyles ST. The neighborhood social environment and physical activity: a systematic scoping review. Int J Behav Nutr Phy. 2019;16(1):124. DOI: 10.1186/s12966-019-08737.

23. Zenk SN, Schulz AJ, Matthews SA, Odoms-Young A, Wilbur J, Wegrzyn L, et al. Activity space environment and dietary and physical activity behaviors: A pilot study. Health Place. 2011;17(5):1150-61.

DOI: 10.1016/j.healthplace.2011.05.001.

24. Zhou P, Grady SC, Chen G. How the built environment affects change in older people's physical activity: A mixed-methods approach using longitudinal health survey data in urban China. Soc Sci Med. 2017;192:74-84. DOI: 10.1016/j.socscimed.2017.09.032.

25. Wilcox S, Castro C, King AC, Housemann R, Brownson RC. Determinants of leisure time physical activity in rural compared with urban older and ethnically diverse women in the United States. J Epidemiol Community Health. 2000;54(9):667-72. DOI: 10.1136/jech.54.9.667.

26. González S, Sarmiento OL, Lozano O, Ramírez A, Grijalba C. Niveles de actividad física de la población colombiana: desigualdades por sexo y condición socioeconómica.
Biomédica. 2014;34(3):447-59.

DOI: 10.7705/biomedica.v34i3.2258.

27. Vélez-Álvarez C, Vidarte Claros JA, Parra Sánchez JH. Niveles de sedentarismo en población entre 18 y 60 años en Manizales, Pereira y Armenia, Colombia. Análisis Multivariado. Aquichan. 2014;14(3):303-15. DOI: 10.5294/aqui.2014.14.3.3.

28. Quiroz-Mora CA, Serrato Ramírez DM, Bergonzoli Peláez G. Factors associated with adherence to physical activity in patients with chronic non-communicable diseases. Rev Salud Pública. 2018;20(4):460-4. DOI: 10.15446/rsap.v20n4.62959.

29. Cleland C, Ferguson S, Ellis G, Hunter RF. Validity of the International Physical Activity Questionnaire (IPAQ) for assessing moderate-to-vigorous physical activity and sedentary behaviour of older adults in the United Kingdom. BMC Med Res Methodol. 2018;18(1):176. DOI: 10.1186/s12874-018-0642-3.

30. Brugnara L, Murillo S, Novials A, Rojo-Martínez G, Soriguer F, Goday A, et al. Low Physical Activity and Its Association with Diabetes and Other Cardiovascular Risk Factors: A Nationwide, Population-Based Study. PLoS ONE. 2016;11(8):e0160959. 10.1371\%2Fjournal.pone.0160959.

31. Shi L, Shu X-O, Li H, Cai H, Liu Q, Zheng W, et al. Physical Activity, Smoking, and Alcohol Consumption in Association with Incidence of Type 2 Diabetes among Middle-Aged and Elderly Chinese Men. PLoS ONE. 2013;8(11):e77919. DOI: 10.1371\%2Fjournal.pone.0077919.

32. Lehne G, Voelcker-Rehage C, Meyer J, Bammann K, Gansefort D, Brüchert T, Bolte G. Equity Impact Assessment of Interventions to Promote Physical Activity among Older Adults: A Logic Model Framework. International journal of environmental research and public health. 2019;16(3):420. DOI: 10.3390/ijerph16030420.

33. Gallardo Solarte K, Benavides Acosta FP, Rosales Jiménez R. Costos de la enfermedad crónica no transmisible: la realidad colombiana. Rev Cienc Salud. 2016;14(1):103-14. DOI: 10.12804/revsalud14.01.2016.09. 